\title{
Unidades de paisaje locales en el noreste de Michoacán, México
}

\section{Local landscape units in the northeast of Michoacán, México}

\author{
Alexis López-Navarro \\ Genaro Aguilar-Sánchez ${ }^{2}$ \\ Universidad Autónoma Chapingo, México
}

\begin{abstract}
Resumen
El objetivo fue elaborar un mapa de unidades de paisaje combinando un enfoque experto y local, para la planeación del territorio en el Noreste del Estado de Michoacán. El enfoque experto uso la metodología de los paisajes físico-geográficos, obteniendo unidades del paisaje, las cuales fueron interpretadas por los actores locales, mediante la aplicación de herramientas participativas; donde se aplicaron entrevistas semiestructuradas, transectos en campo; se realizaron mapas mentales, y se emplearon los Sistemas de Información Geográficos. Se obtuvo que ambos enfoques tienen diferencias contrastantes. Por un lado, el enfoque experto define las unidades de paisajes por rasgos biofísicos; en cambio, las personas mediante el conocimiento que tienen, reconocen los atributos y delimitan las unidades de paisaje, por los usos del suelo que les dan. Se concluye, que es importante del uso de métodos híbridos para el estudio de las prácticas comunitarias en el manejo de los recursos de uso común.
\end{abstract}

Palabras clave: Planeación participativa, Conocimiento local, Paisajes, Sistemas de Información Geográficos Participativos.

1 MC. Universidad Autónoma Chapingo, 56230, Texcoco, Estado de México. Correo electrónico: alp_n@hotmail.com

2 DR. Universidad Autónoma Chapingo, 56230, Texcoco, Estado de México. Correo electrónico: g_aguila@correo.chapingo.mx

Este artículo corresponde a la ponencia presentada en el 35th Conference of Latin American Geographers realizada en San José, Costa Rica del 20 al 22 de mayo del 2018. 


\begin{abstract}
The objective was to prepare a map of landscape units combining an expert and local approach, for the planning of the territory in the Northeast of the State of Michoacán. The expert approach used the methodology of physical-geographical landscapes, obtaining landscape units, which were interpreted by local actors, through the application of participatory tools; where semi-structured interviews were applied, transects in the field; mental maps were made, and Geographical Information Systems were used. It was found that both approaches have contrasting differences. On the one hand, the expert approach defines the units of landscapes by biophysical features; instead, people through the knowledge they have, recognize the attributes and delimit the landscape units, by the land uses that they give them. It is concluded that it is important to use hybrid methods to study community practices in the management of common use resources.
\end{abstract}

Keywords: Participatory Spatial Planning, Local knowledge, landscapes, Participatory Geographic Information Systems.

\title{
Introducción
}

En México, las actividades agropecuarias y forestales son esenciales para el sector rural, debido a que son las principales fuentes de ingresos y son el medio de subsistencia para su población. Sin embargo, sin el manejo adecuado, éstas pueden generar impactos negativos al ambiente por el mal uso del agua, la deforestación, el uso excesivo de plaguicidas, entre otras (Escalante, 2006). Situación que representa uno de los enormes retos en la estrategia del desarrollo del país. Para superar los problemas ambientales acotados el Estado ha establecido políticas públicas y reformas jurídicas instrumentadas mediante leyes, organismos públicos y modelos de planeación territorial a diferentes escalas (nacional, regional y local) (Negrete \& Aguilar, 2006; Ojeda et al. 2015).

En el modelo de planeación territorial local está contenido el Ordenamiento Territorial Comunitario (OTC), el cual es considerado un instrumento dirigido a la organización y planificación de actividades silvícolas en los espacios de uso común de los núcleos agrarios (CONAFOR, 2007). Éste tiene como principio reconocer que las comunidades rurales poseen una importante experiencia en la administración de los recursos naturales (Negrete \& Bocco, 2003). Uno de los requerimientos metodológicos en los OTC, es la integración de unidades de paisaje en las que se describan las características cualitativas y cuantitativas, respecto las condiciones socioeconómicas y ambientales del territorio estudiado (CONAFOR, 2007).

No obstante que éste modelo de planificación territorial se asume local y participativo, una práctica común en la integración unidades de 
paisajes es el uso de criterios y métodos convencionales provenientes de la geomorfología, suelos, drenaje fluvial y cobertura vegetal (Bocco et al., 1999; Bocco et al., 2001; González et al., 2009; Soto, 2010). Esto evidencia la falta de articulación con el conocimiento empírico de los usuarios directos de los recursos naturales, donde se reflejen los entendimientos locales en un contexto cartográfico (Fagerholm et al., 2013; Pájaro, 2010). En base a lo anterior se realizó un mapa de unidades de paisaje combinando un enfoque experto y local, para la planeación del territorio en el Noreste del Estado de Michoacán.

\section{Localización y características generales del área de estudio}

La investigación se realizó en el ejido de Santa Cruz Ojo de Agua, el cual se ubica al Noroeste del municipio de Epitacio Huerta, Michoacán, entre las coordenadas UTM-14Q: 2, 233,000 y 2, 239,000 metros en coordenadas Norte; 359,300 y 368,500 metros en coordenadas Oeste. Tiene sus colindancias al norte con la localidad de San Cristóbal; al sur con la Palos Altos; al este con las de Astilleros y La Salud; al oeste con propiedad la privada de Velázquez y el Rancho de Manuel Ruiz (Figura 1).

El ejido fue creado en resolución presidencial el día 2 de Agosto de 1950 y se reporta que tiene una superficie total de $1,231.5196$ ha con un total de 136 ejidatarios, 61 posesionarios y 33 avecindados. Actualmente, está constituido por una asamblea y cuenta con sus órganos de representación estipulados por la ley agraria vigente. 
Figura 1. Localización del área de estudio

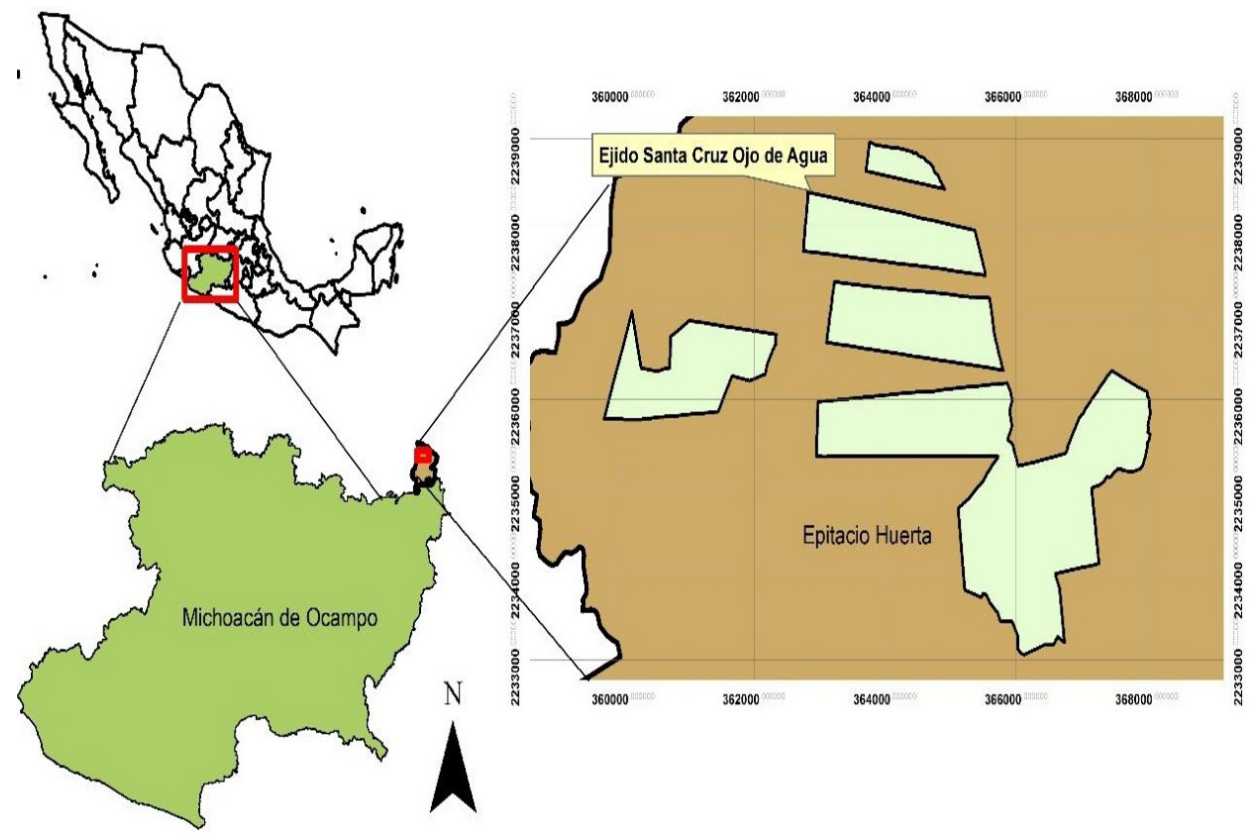

Fuente: Elaborado a partir de datos vectoriales, escala 1:50000, INEGI, 2015

El área de estudio se encuentra una zona con alturas que van desde los 2400 a $2700 \mathrm{msnm}$, en un clima C(W1)(W), caracterizado como templado subhúmedo con lluvias en verano y con un porcentaje de lluvia invernal menor de 5 (García, 2004).

Existen zonas de pastoreo extensivo con pastizal natural en 567.55 ha, lo que representa un $46 \%$ del total de su superficie; seguida por un uso agrícola con maíz de temporal en una extensión de 424.32 ha, representando un $34.5 \%$ de su superficie total; así también, el uso forestal se caracteriza por una vegetación arbórea de latifoliadas con elementos de encino (Quercus sp.), y de encinos asociados con chaparral, los cuales se concentran en 239.56 ha (19.5\% de la superficie total del ejido). Por último, en una porción menor del ejido existe una asociación de vegetación de matorral subinerme con pastizal natural (INEGI, 1974).

Entre los principales aspectos sociales y económicos se reporta que existen 820 habitantes, la mayoría de ellos han estudiado hasta la escuela primaria, y en la actualidad no se cuenta con registros de que se hable alguna 
lengua original dentro del ejido. Las principales fuentes de ingresos son por actividades agrícolas y ganaderas, así como el trabajo extracomunitario, principalmente, por la emigración de jóvenes hacia la cabecera municipal de Epitacio Huerta o a la ciudad de Querétaro (AAPYREFO, 2014).

\section{Marco Teórico conceptual}

El estudio del paisaje desde el punto de vista de las escuelas europeas ha sido retomado en América Latina por las escuelas de geografía de cuba y Brasil, las que han estudiado el paisaje para adecuar los principios y métodos a las condiciones de los países considerados de Tercer Mundo (Mateo \& da Silva, 2007; Salinas, 2005). También, la conceptualización del paisaje ha tenido transformaciones a medida que se ha interrelacionado con otras disciplinas científicas considerándose como un sistema de conceptos, lo cual quiere decir que se ha transformado en la medida que la sociedad interacciona con su medio natural, y es por eso que el paisaje se reconoce mediante varias acepciones, entre las cuales se destacan: paisaje natural, el paisaje antropo-natural, paisaje cultural, y el paisaje visual o percibido (Flores, 2010).

Chiappy, et al. (2000), mencionan que el concepto de paisaje ha sido muy discutido y controvertido en los últimos años debido a la jerarquía taxonómica que se asigna a uno o varios de sus componentes, a su extensión o cobertura y a la temporalidad con la que se conciban estos. A pesar que no existe una definición que satisfaga completamente a todos los puntos de vista, es por eso que en la literatura actual aparecen definiciones que tienden a concatenar los componentes físicos y bióticos que conforman el medio natural. Por lo anterior, en consideración de la conceptualización del paisaje para este trabajo de investigación se acota a lo que la escuela cubana de geografía define, Mateo (1984), concibe a los paisajes como sistemas territoriales integrados por componentes naturales y complejos de diferentes rangos taxonómicos, formados bajo la influencia de los procesos naturales y de la actividad modificadora de la sociedad humana, que se encuentra en permanente interacción y se desarrolla históricamente.

La interpretación de las características del suelo, tierra, hecha por los productores es muy importante como lo reportan en otros casos de estudios relacionados a la etnoedafología en el estado de Michoacán (Barrera, 1998; Pájaro, 2010; Barajas, 2012; Pulido \& Bocco, 2016). Es importante 
tener claros los conceptos ya que en el trabajo se emplea una metodología mixta, donde se integra el enfoque de los paisajes físico-geográficos con el conocimiento local de los ejidatarios para mejorar tales unidades de paisaje, mediante la aplicación de herramientas cualitativas y actividades de mapeo participativo para producir un insumo cartográfico de zonificación definitivo.

Para sustentar la utilización de este enfoque mixto, se consideró el análisis que Barrera (2009) hace de la definición de espacio con base en lo postulado por Lefebvre en 1974 el que menciona

"el análisis y la representación de los espacios vividos y concebidos, debe hacerse con base en métodos cualitativos, en los cuales se analizan otros aspectos del espacio, que junto con el espacio material o cartesiano, constituyen la trilogía del espacio, mediante la cual debe ser posible tener un mayor acercamiento y entendimiento del mundo real"

Con la idea de crear un puente de comunicación entre las representaciones euclidianas de un mapa convencional o experto y las estructuradas por el conocimiento local.

\section{Marco Metodológico}

Para la presente investigación se empleó el enfoque multimétodo propuesto por Aguirre (2010), quien realizó un análisis comparativo de las unidades de paisaje generada por un enfoque experto con el conocimiento campesino del paisaje en el ejido Nexpa, Michoacán. Así entonces, el marco metodológico para este estudio se compone de tres etapas. En la primera se elaboró un mapa de unidades de paisaje físico-geográficas, en la segunda los ejidatarios del área de estudio identificaron e interpretaron sus propias unidades de paisaje con base al mapa elaborado en la primera etapa mencionada y finalmente se analizaron las diferencias de las unidades cartográficas de ambos enfoques mediante la prueba estadística de la CHI-CUADRADA y el cálculo del porcentaje de correspondencia espacial por unidad cartográfica (Figura 2). 
Figura 2. Marco metodológico de la investigación

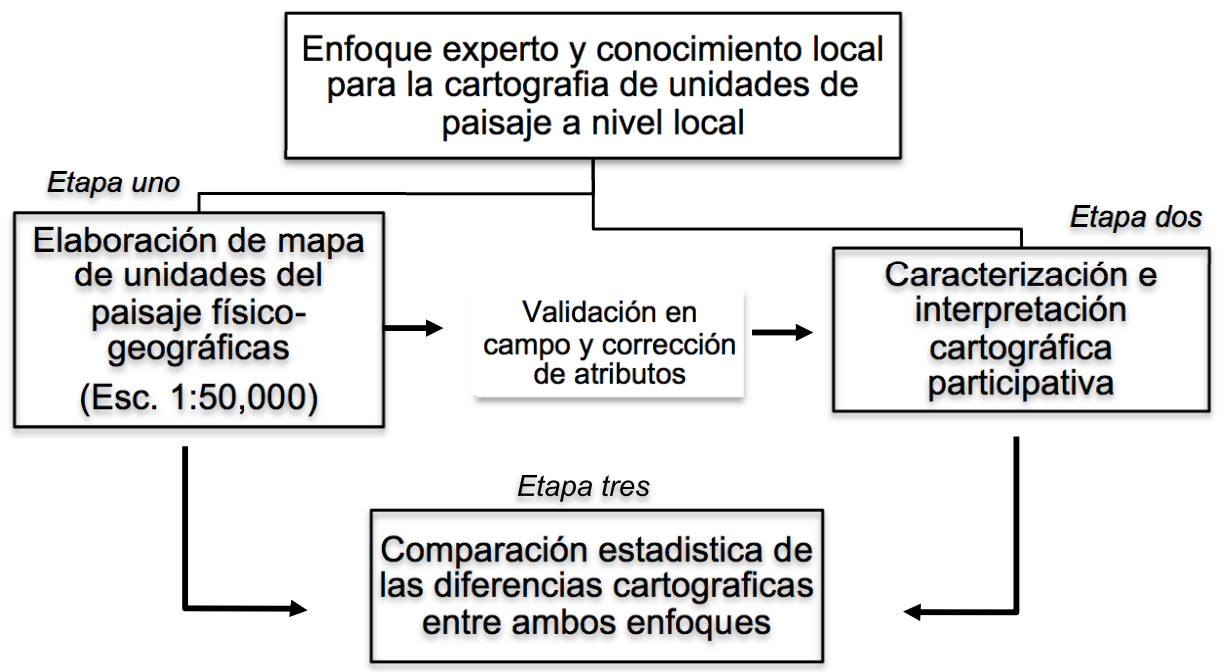

Fuente: Elaboración propia basado en Aguirre (2010)

Elaboración de mapa de unidades del paisaje físico-geográficas

Para esta primera fase del trabajo se tomó en cuenta la metodología para la generación semiautomatizada de unidades de paisaje de Priego et al., (2010), y también la clasificación taxonómica del paisaje basado en el esquema integral de componentes naturales definido por Mateo (2002), donde se identifican tres unidades tipológicas del paisaje (localidades, parajes complejos y parajes simples). Para lo anterior, se procesó la información cartográfica útil para esta etapa metodológica con el software ArcGIS 10 (Cuadro 2). 
Cuadro 1. Insumos cartográficos usados para la construcción del mapa de paisajes físicos-geográficos

\begin{tabular}{|l|l|l|}
\hline \multicolumn{1}{|c|}{ Insumo cartográfico } & \multicolumn{1}{|c|}{ Características } & \multicolumn{1}{c|}{ Fuente } \\
\hline $\begin{array}{l}\text { Modelo Digital de } \\
\text { Clima }\end{array}$ & $\begin{array}{l}\text { Producto raster con resolución } \\
\text { de } 15 \text { metros. }\end{array}$ & $\begin{array}{l}\text { Continuo de elevación } \\
\text { Mexicano (CEM 3.0) del } \\
\text { INEGI, 2013. }\end{array}$ \\
\hline Geología & $\begin{array}{l}\text { Producto vectorial con escala } \\
1: 1000 \text { 000. }\end{array}$ & INEGI, 2008. \\
\hline $\begin{array}{l}\text { Carta temática con clave } \\
\text { F14C85 Jerécuaro-Presa Solís } \\
\text { y F14C86 Amealco, escala } \\
1: 50 \text { 000. }\end{array}$ & $\begin{array}{l}\text { Comisión de Estudios } \\
\text { del Territorio Nacional } \\
\text { (CETENAL), ahora INEGI, } \\
\text { 1973. }\end{array}$ \\
\hline $\begin{array}{l}\text { Carta temática con clave } \\
\text { F14C85 Jerécuaro-Presa Solís } \\
\text { y F14C86 Amealco, escala } \\
\text { 1:50 000. }\end{array}$ & $\begin{array}{l}\text { Comisión de Estudios } \\
\text { del Territorio Nacional } \\
\text { (CETENAL), ahora INEGI, } \\
\text { 1974. }\end{array}$ \\
\hline Cagetación & $\begin{array}{l}\text { Carta temática con clave } \\
\text { F14C85 Jerécuaro-Presa Solís } \\
\text { y F14C86 Amealco, escala } \\
\text { 1:50 000. }\end{array}$ & $\begin{array}{l}\text { Comisión de Estudios } \\
\text { del Territorio Nacional } \\
\text { (CETENAL), ahora INEGI, } \\
\text { 1974. }\end{array}$ \\
\hline $\begin{array}{l}\text { Catos vectoriales de las cartas } \\
\text { con clave F14C85 Jerécuaro- } \\
\text { Presa Solís y F14C86 } \\
\text { Amealco, escala 1:50 000. }\end{array}$ & INEGI, 2015. \\
\hline
\end{tabular}

Fuente: elaboración propia con base en INEGI, 1973, 1974, 2008, 2015.

De acuerdo a lo establecido en la metodología mencionada, las unidades de paisaje se construyeron de lo general a lo particular y se determinaron a escala 1:50,000, por lo que fue importante considerar el axioma del Área Mínima Cartografiable (AMC) para realizar una generalización espacial; dicha generalización requiere identificar el conjunto de identidades geográficas menores a $40000 \mathrm{~m}^{2}$ de superficie ( 4 x $4 \mathrm{~mm}$ en un mapa impreso) y asignarlas a los polígonos contiguos que contengan mayor superficie, es decir, que los mapas generados en cada secuencia metodológica quedaron conformados de polígonos con una superficie mayor de $40000 \mathrm{~m}^{2}$.

En la Figura 3, se observa el flujograma que resume la secuencia metodológica. Como primer paso se obtuvieron las unidades superiores del paisaje denominadas localidades, después los parajes complejos y los parajes simples de la siguiente manera: 
Figura 3. Flujograma para la construcción del mapa de paisajes físico-geográficos

Mapa de unidades de
paisaje escala 1:50,000

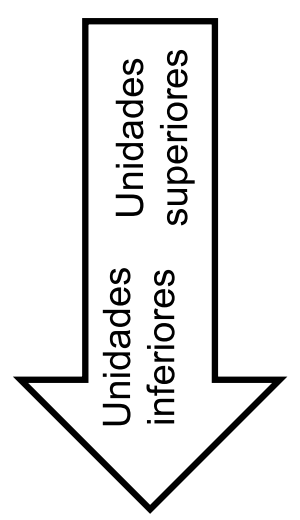

Localidades $\quad \begin{gathered}\text { Disección vertical } \\ +\begin{array}{c}\text { Litología } \\ \text { Clima }\end{array}\end{gathered} \quad I, I I, I I I, \ldots$

\begin{tabular}{|c|cc}
\hline \multirow{2}{*}{$\begin{array}{c}\text { Parajes } \\
\text { complejos }\end{array}$} & $\begin{array}{c}\text { Localidades }+ \\
\text { Mesoformas } \\
\text { del relieve }\end{array}$ & I.1, II.1, \\
\cline { 2 - 3 } & & $\ldots$
\end{tabular}

Fuente: elaboración propia, a partir de Priego et al., (2010).

A continuación, se llevó a cabo la validación en campo y corrección de posibles errores en los atributos de las unidades de paisaje físico-geográficas con la participación de las autoridades ejidales, quienes organizados en equipos visualizaron el mapa impreso, y definieron la ruta a seguir para realizar el recorrido de las unidades de paisaje y los parajes delimitados en el mapa hecho gabinete, lo cual se hizo por transectos. En el recorrido se consideró el número de sitios necesarios para la toma de datos de acuerdo a la no correspondencia de los atributos del mapa en el terreno. Se validó información referente a la geoforma, la pendiente del terreno, la geología, la unidad de suelo, el tipo de cobertura y el uso del suelo. Como instrumentos de medición se utilizaron un clinómetro pm5 Suunto, GPSMap62 Garmin, pala y cámara fotográfica.

\section{Caracterización e interpretación cartográfica participativa}

En la realización de la siguiente etapa metodológica, se trabajó en dos sesiones de talleres participativos con integrantes de la asamblea ejidal, de los cuales únicamente colaboraron quince personas ejidatarias de las tres zonas urbanas que componen al ejido (Tepozán, Santa Cruz Ojo de Agua y Cerrito). Se formaron equipos compuestos de personas que 
habitaran la misma localidad, y mediante la técnica de mapa mental dibujaron los elementos de los paisajes de su entorno, con el proceso lógico con el que se construyeron las unidades de paisaje en el método experto; es decir, los lugareños dibujaron los elementos constituyentes del paisaje de lo general a lo particular. En seguida, se trabajó sobre el mapa impreso de las unidades hechas en gabinete, donde los ejidatarios reconocieron y delimitaron las que pudieron observar en su ejido. Por último, se sintetizó la información de la delimitación y caracterización de las unidades de paisaje, las cuales fueron digitalizadas en gabinete con el software ArcGIS 10, para obtener un mapa reconstruido de los paisajes físico-geográficos integrando el conocimiento local de los ejidatarios.

Comparación estadística de las diferencias cartográficas entre ambos enfoques

Para la realización de este apartado, como variable cuantitativa fue tomada en cuenta la frecuencia y la correspondencia especial de las de unidades de paisaje en ambos enfoques. Para la primera se utilizó la prueba de bondad de ajuste que mide la correspondencia entre la frecuencia observada de polígonos de las unidades de paisaje interpretada por las personas con la frecuencia esperada de las mismas desde el enfoque físico-geográfico. La prueba estadística usada es conocida como CHI-CUADRADA, la que se define como sigue:

$$
X^{2}=\sum_{i=1}^{K} \frac{(O i-E i)^{2}}{E i}
$$

Donde:

$O i=$ es la frecuencia observada en cada categoría de unidad de paisaje $E i=$ es la frecuencia esperada en cada categoría de unidad de paisaje $\sum=$ es la sumatoria total de las categorías de unidades del paisaje

El valor calculado de $X^{2}$ fue comparado con un valor crítico de distribución $\left(X_{(t)}^{2}\right)$, para poder hacer la prueba de decisión; No se rechaza hipótesis nula $\left(\mathrm{H}_{\mathrm{o}}\right)(\mathrm{La}$ frecuencia esperada es la mima que la frecuencia observada en los datos) cuando el valor de $X^{2}<X^{2}{ }_{(t)}$, en caso contrario si se rechaza. Para ello se buscó el valor de tablas con 6 grados de libertad y un 
P-valor de 0.1. Aunado a lo anterior, se calculó el porcentaje de correspondencia espacial, donde se obtuvieron las superficies de cada categoría de paisaje del enfoque experto y se comparó con la superficie de los polígonos de cada unidad de paisaje interpretado por los lugareños.

\section{Resultados y Discusión}

Los resultados de investigación obtenidos se dividieron en tres apartados. En el primero, se muestran el número de unidades cartográficas y los factores de diferenciación de las unidades de paisaje del mapa físicogeográfico (enfoque experto); en el segundo apartado, se presentan los resultados referentes a la diferenciación de los atributos y la delimitación de los paisajes por el conocimiento local a partir del primer resultado y por último, se muestra la bondad de ajuste estadístico entre las unidades de paisaje de ambos enfoques, así como el porcentaje de correspondencia espacial entre éstas.

-Unidades del paisaje físico-geográficas del ejido de Santa Cruz Ojo de Agua, Epitacio Huerta, Michoacán

Con la metodología propuesta por Priego et al., (2010). Se obtuvieron las unidades de paisaje físico-geográficos, a escala 1:50,000, teniendo como resultado siete localidades, catorce parajes complejos y veintiocho parajes simples (Figura 4).

La localidad V es la unidad de paisaje que más superficie ocupa en todo el ejido con una superficie de $5.49 \mathrm{~km}^{2}(46.6 \%)$, ésta se caracteriza por estar en un sistema de planicies con pendientes que oscilan de $1^{\circ}$ a $10^{\circ}$, con rocas ígneas extrusivas y suelos Phaeozem háplico, utilizado para la agricultura de temporal. Dentro de esta unidad de paisaje se encuentran diferenciados dos parajes complejos y cuatro parajes simples. La demás localidades son menores. Las demás localidades se detallan en el cuadro (3) 
Alexis López-Navarro, Genaro Aguilar-Sánchez. Local landscape units in the northeast of Michoacán, México

Figura 4. Mapa de paisajes físico-geográficos del ejido Santa Cruz Ojo de Agua, Epitacio Huerta, Michoacán.

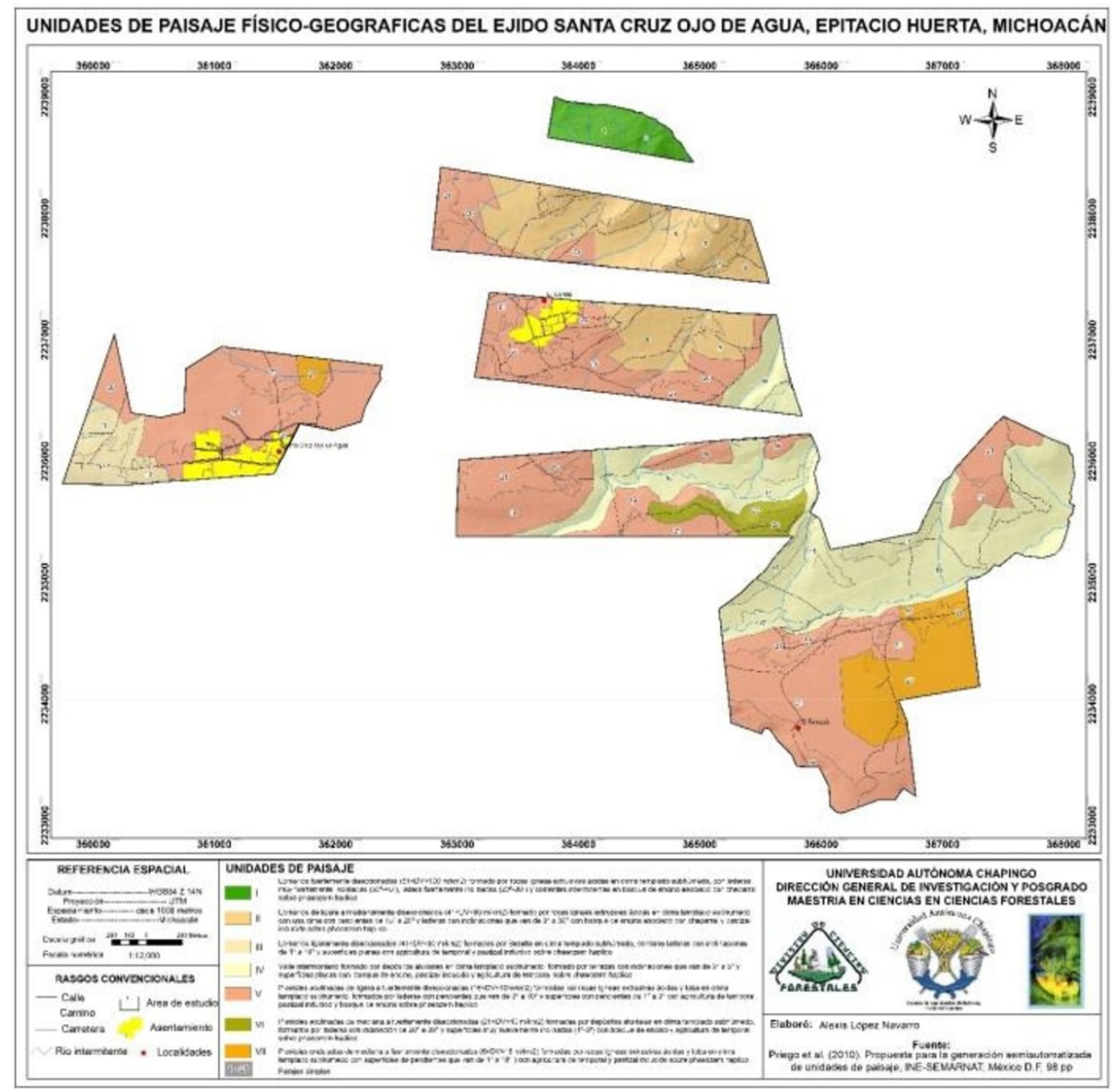

Fuente: Elaboración propia con base en la metodología de Priego et al., 2010 
Cuadro 3. Características biofísicas de las unidades de paisaje físico-geográficas

\begin{tabular}{|c|c|c|c|c|c|c|c|c|}
\hline \multirow{2}{*}{ Loc } & \multirow{2}{*}{ Pc } & \multirow{2}{*}{ Ps } & \multirow{2}{*}{ Geoforma } & \multirow{2}{*}{ Litología } & \multirow{2}{*}{ Suelos } & \multirow{2}{*}{$\begin{array}{c}\text { Vegetación y uso del } \\
\text { suelo }\end{array}$} & \multicolumn{2}{|c|}{ Superficie } \\
\hline & & & & & & & $\mathbf{K m}^{2}$ & $\%$ \\
\hline I & 2 & 3 & Lomerío & Igea & Litosol & $\begin{array}{l}\text { Bosque de encino y } \\
\text { chaparral }\end{array}$ & 0.35 & 2.8 \\
\hline II & 3 & 7 & \begin{tabular}{|l|} 
Lomerío \\
\end{tabular} & Igea & $\begin{array}{l}\text { Phaeozem } \\
\text { háplico + } \\
\text { Phaeozem } \\
\text { lúvico }\end{array}$ & $\begin{array}{l}\text { Bosque de encino, } \\
\text { chaparral y pastizal }\end{array}$ & 1.74 & 14.1 \\
\hline III & 2 & 4 & \begin{tabular}{|l|} 
Lomerío \\
\end{tabular} & Basalto & \begin{tabular}{|l|}
$\begin{array}{l}\text { Phaeozem } \\
\text { háplico }\end{array}$ \\
\end{tabular} & $\begin{array}{l}\text { Pastizal y agricultura } \\
\text { de temporal }\end{array}$ & 0.37 & 3 \\
\hline IV & 2 & 5 & Valle & \begin{tabular}{|l} 
Deposito \\
aluvial
\end{tabular} & \begin{tabular}{|l|} 
Phaeozem \\
háplico + \\
Phaeozem \\
lúvico \\
\end{tabular} & $\begin{array}{l}\text { Bosque de encino, } \\
\text { pastizal y agricultura } \\
\text { de temporal }\end{array}$ & 3.06 & 24.8 \\
\hline $\mathrm{V}$ & 2 & 4 & Planicie & Igea, Toba & $\begin{array}{l}\text { Phaeozem } \\
\text { háplico }\end{array}$ & $\begin{array}{l}\text { Agricultura de } \\
\text { temporal, pastizal, } \\
\text { chaparral y bosque de } \\
\text { encino }\end{array}$ & 5.69 & 46.2 \\
\hline VI & 2 & 2 & Planicie & $\begin{array}{l}\text { Deposito } \\
\text { aluvial }\end{array}$ & $\begin{array}{l}\text { Phaeozem } \\
\text { háplico }\end{array}$ & $\begin{array}{l}\text { Bosque de encino } \\
\text { y agricultura de } \\
\text { temporal }\end{array}$ & 0.24 & 1.9 \\
\hline \multirow[t]{2}{*}{ VII } & 1 & 4 & Planicie & Igea, Toba & $\begin{array}{l}\text { Phaeozem } \\
\text { háplico }\end{array}$ & $\begin{array}{l}\text { Pastizal y agricultura } \\
\text { de temporal }\end{array}$ & 0.86 & 7 \\
\hline & & & & & \multicolumn{2}{|l|}{ Total } & 12.31 & 100 \\
\hline
\end{tabular}

Loc: Localidad, Pc: Parajes complejos, Ps: Parajes simples, Igea: Ígnea extrusiva ácida.

Fuente: Elaboración propia con base al mapa de unidades físico-geográficas.

Derivado de los resultados se pudo observar que las localidades fueron diferenciadas por la geoforma y las diferentes clases litológicas, donde se obtuvieron paisajes caracterizados por sistemas de lomeríos, valles y planicies, siendo las planicies las que se encuentran en mayor proporción en el paisaje del ejido, seguidas por valles intermontanos y lomeríos. Así mismo, para los paisajes en su forma más detallada, los factores de diferenciación fueron las mesoformas del relieve como son barrancos, laderas, terrazas, colinas y superficies, además, por las pendientes del terreno. Estas características fueron las que condicionaron la existencia de los parajes complejos y los parajes simples, respectivamente.

Los factores mencionados fueron semejantes con los resultados que otros autores como Aguirre (2010), Sánchez (2011) y Espinoza (2013), han reportado al utilizar dicho método en gabinete. 
-Caracterización e interpretación cartográfica de unidades del paisaje participativa

Derivado de la actividad de mapa mental y de transectos en campo, se obtuvo la caracterización de los paisajes del área de estudio (Cuadro 4). Como se pudo observar los ejidatarios al interpretar los rasgos del relieve caso concreto al lomerío y planicie, los definieron con el nombre de cerro y plano respectivamente; y para el caso de los valles, éstos no pudieron reconocerlos; sin embargo, distinguieron dos atributos dentro de éstos. Por un lado reconocieron como quebradas a las terrazas, y por otro, a las superficies planas como arroyos. De la misma manera, como parte de las características topográficas las pendientes en el terreno fueron asociadas con dos aspectos: primero por su forma, a la cual definieron como plano, en referencia a las que tienen poca pendiente; y onduladas a las que tienen una pendiente que oscila entre $5^{\circ}$ a $10^{\circ}$; y segundo, a las que tienen mayor pendiente, las denominaron terreno cuesta arriba. Esto coincide con el estudio realizado por Pulido y Bocco (2010), quienes documentan el conocimiento de una comunidad indígena purépecha, donde existen palabras similares para denominar algunas formas del terreno.

Las características de los suelos descritos por los ejidatarios del área de estudio, son muy similares a las que se reportadas en otros casos de estudios relacionados a la etnoedafología en el estado de Michoacán (Barrera, 1998; Barajas, 2012; Pulido \& Bocco, 2016). 
Cuadro 4. Atributos del paisaje de acuerdo al conocimiento local

\begin{tabular}{|l|l|l|l|}
\hline \multicolumn{2}{|l|}{ Atributo experto } & \multicolumn{1}{c|}{ Atributo local } \\
\hline \multirow{4}{*}{ Geoforma } & Lomerío & Cerro \\
\cline { 2 - 3 } & \multirow{2}{*}{ Valle } & Terrazas & Quebradas \\
\cline { 2 - 3 } & Planicie & Superficies planas & Arroyos \\
\hline \multirow{4}{*}{ Litología } & Ígnea extrusiva ácida & Plano \\
\cline { 2 - 3 } & Toba & Risco \\
\cline { 2 - 3 } & Basalto & Cantera \\
\cline { 2 - 3 } & Deposito aluvial & Maciza \\
\hline \multirow{4}{*}{ Pendiente } & $1^{\circ}-3^{\circ}$ & Tierra barrosa \\
\cline { 2 - 3 } & $3^{\circ}-10^{\circ}$ & Casi Plano \\
\cline { 2 - 3 } & $10^{\circ}-45^{\circ}$ & Ondulado \\
\hline \multirow{3}{*}{ Uso de suelo } & Agrícola & Cuesta arriba \\
\cline { 2 - 3 } & Pecuario & Tierras de labor \\
\cline { 2 - 3 } & Forestal & Tierras de uso común \\
\hline \multirow{2}{*}{ Vegetación } & Bosque de encino & Bosque encino y pino \\
\cline { 2 - 3 } & Chaparral & Plantas de uso medicinal \\
\hline \multirow{3}{*}{ Suelos } & Phaeozem lúvico & Tierra colorada \\
\cline { 2 - 3 } & Phaeozem háplico & Tierra blanca \\
\cline { 2 - 3 } & Litosol & Tierra negra/barrosa \\
\hline
\end{tabular}

Fuente: Elaboración propia con base a la interpretación de los ejidatarios

Finalmente, las unidades de paisaje que los ejidatarios definieron con base al mapa físico-geográfico, fueron en total dieciocho (Figura 5), donde se observa que los criterios utilizados por los actores locales para delimitarlas, estuvieron dados mayormente por el uso que cotidianamente les dan a las diferentes áreas de su territorio, asignándoles distintas atribuciones de acuerdo a diversas prácticas que en la actualidad realizan y otras que han dejado de hacer, pero que revelan el significado cultural en el contexto de la comunidad. Así mismo del total identificadas, únicamente tres unidades de paisaje fueron identificadas por la forma del terreno (Cuadro 5) 
Figura 5. Mapa de unidades de paisaje basado en el conocimiento local

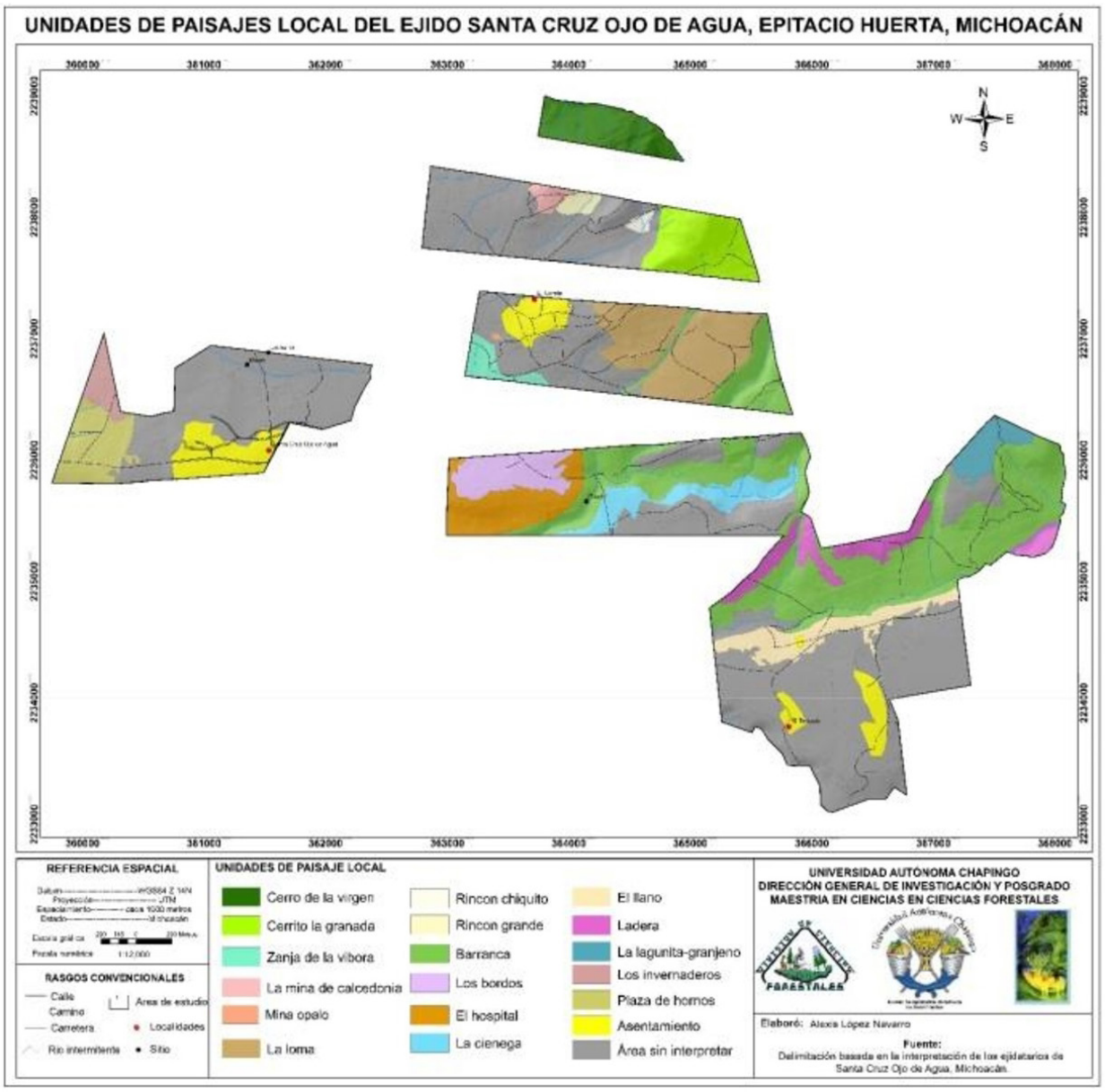

Fuente: Elaboración propia, basada en la interpretación de las unidades de paisaje de los ejidatarios de Santa Cruz Ojo de Agua 
Cuadro 5. Caracterización local de las unidades de paisaje.

\begin{tabular}{|c|c|}
\hline Unidad de paisaje local & Características \\
\hline El cerro de la virgen & Denominado así por ser la zona mejor conservada del ejido \\
\hline Cerrito la granada & $\begin{array}{l}\text { Llamado así por la abundancia de la planta pingüica } \\
\text { (Arctostaphylos sp.) }\end{array}$ \\
\hline Zanja de la víbora & $\begin{array}{l}\text { Se le denomina de así por la forma que asemeja a ese } \\
\text { reptil, es un canal que se sitúa en el lado sur-oeste de la } \\
\text { población de El Cerrito }\end{array}$ \\
\hline Mina de calcedonia & $\begin{array}{l}\text { Sitio en abandono ubicado en las inmediaciones del cerro } \\
\text { de la virgen }\end{array}$ \\
\hline Mina de ópalo & $\begin{array}{l}\text { Sitio en abandono ubicado cerca de la zona de } \\
\text { asentamiento humano de la localidad del cerrito }\end{array}$ \\
\hline La loma & $\begin{array}{l}\text { Es un sitio con pendientes onduladas a cuesta arriba, } \\
\text { dedica a tierras de labor }\end{array}$ \\
\hline Rincón grande & Es una zona que está en las faldas del cerro de la virgen \\
\hline Rincón chiquito & $\begin{array}{l}\text { Es una zona paralela a rincón grande, pero es más pequeña } \\
\text { en tamaño }\end{array}$ \\
\hline Barranca & Sitio denominado al área del cauce de los arroyos \\
\hline Los bordos & Llamado así por la existencia de bordos \\
\hline EI hospital & En el pasado era un sitio donde curaban animales \\
\hline La ciénega & Llamado así por la existencia de un manantial \\
\hline EI llano & $\begin{array}{l}\text { Se ubica en la localidad de Tepozán, tiene pendientes } \\
\text { onduladas cercanas a la barranca }\end{array}$ \\
\hline Laderas & $\begin{array}{l}\text { Zonas identificadas con pendiente de cuesta arriba y } \\
\text { bosques de encino }\end{array}$ \\
\hline La lagunita-granjeno & $\begin{array}{l}\text { Es llamado así por el bordo que se encuentra en este sitio y } \\
\text { la planta arbustiva llamada granjeno. }\end{array}$ \\
\hline Los invernaderos & $\begin{array}{l}\text { Llamado así por la existencia de invernaderos en las } \\
\text { inmediaciones con propiedad privada }\end{array}$ \\
\hline Plaza de hornos & $\begin{array}{l}\text { Nombrado así porque en la época de las haciendas, éstas } \\
\text { tenían hornos para hacer carbón }\end{array}$ \\
\hline Asentamiento & Se refiere al centro de población de cada localidad \\
\hline
\end{tabular}

Fuente: Elaboración propia con base al mapa de unidades de paisaje interpretado por los ejidatarios de Santa Cruz Ojo de Agua.

Se puede observar que el enfoque campesino tiende a ser pragmático al hacer una diferenciación utilitaria del paisaje, donde se confirma que la relación hombre-naturaleza existente en diversas culturas, se corresponde de manera armónica. También, resulta evidente que más 
allá de tipificar los paisajes por rasgos físicos, éstos llegan a plasmar los rasgos culturales territoriales que le dan sentido de pertenencia en el mapeo zonal que realizaron. Lo anterior pone en evidencia lo que McCall (2011) dice al respecto de las coincidencias del conocimiento científico y el conocimiento local; afirmando que en muchas ocasiones no existe un punto de coincidencia entre ambos conocimientos al describir los aspectos relevantes de la realidad de un lugar.

-Análisis comparativo entre las unidades de paisaje de ambos enfoques

Los resultados obtenidos en este apartado, fueron posibles a partir de la comparación de las entidades espaciales del mapa físico-geográfico a nivel de localidades y las unidades cartográficas interpretadas por el conocimiento local. Para evaluar el ajuste entre la frecuencia de polígonos de ambos métodos, se encontró que el valor estadístico $\mathrm{X}^{2}$ fue de 11.2 (Cuadro 6).

Cuadro 6. Resultado de la prueba estadística de CHI-CUADRADA para las frecuencias numéricas entre ambos enfoques.

\begin{tabular}{|c|c|c|c|}
\hline Clase & $\begin{array}{c}\text { Frecuencia de polígonos } \\
\text { observada* }\end{array}$ & $\begin{array}{c}\text { Frecuencia de polígonos } \\
\text { esperada** }\end{array}$ & $\mathbf{X}^{\mathbf{2}}$ \\
\hline I & 2 & 2 & 0 \\
\hline II & 8 & 10 & 0.4 \\
\hline III & 2 & 3 & 0.3 \\
\hline IV & 8 & 8 & 0 \\
\hline V & 14 & 27 & 6.3 \\
\hline VI & 0 & 2 & 2.0 \\
\hline VII & 1 & 4 & 2.3 \\
\hline & & $\sum$ & 11.2 \\
\hline
\end{tabular}

* Datos obtenidos del mapa de unidades de paisaje local.

** Datos obtenidos del mapa de unidades de paisaje físico-geográfico.

El resultado anterior se comparó con el valor crítico obtenido en la tabla de distribución de CHI-CUADRADA, el cual fue de 10,6446. Donde se pudo observar que 11.2>10,6446, y de acuerdo con el criterio de decisión se concluye que se rechaza la hipótesis nula, por lo tanto, en términos de unidades cartográficas de paisaje, la frecuencia esperada de los polígonos son estadísticamente distintos a la frecuencia observada de polígonos de las unidades de paisaje. 
Para evaluar las diferencias que se encontraron en las unidades de paisaje, se contabilizó la correspondencia cartográfica de las unidades superiores del paisaje del enfoque experto (localidades) con los polígonos delimitados desde el conocimiento local (Figura 6).

Figura 6. Comparación espacial de las unidades cartográficas producidas con ambos enfoques.

\section{porcentaje de correspondencia espacial}

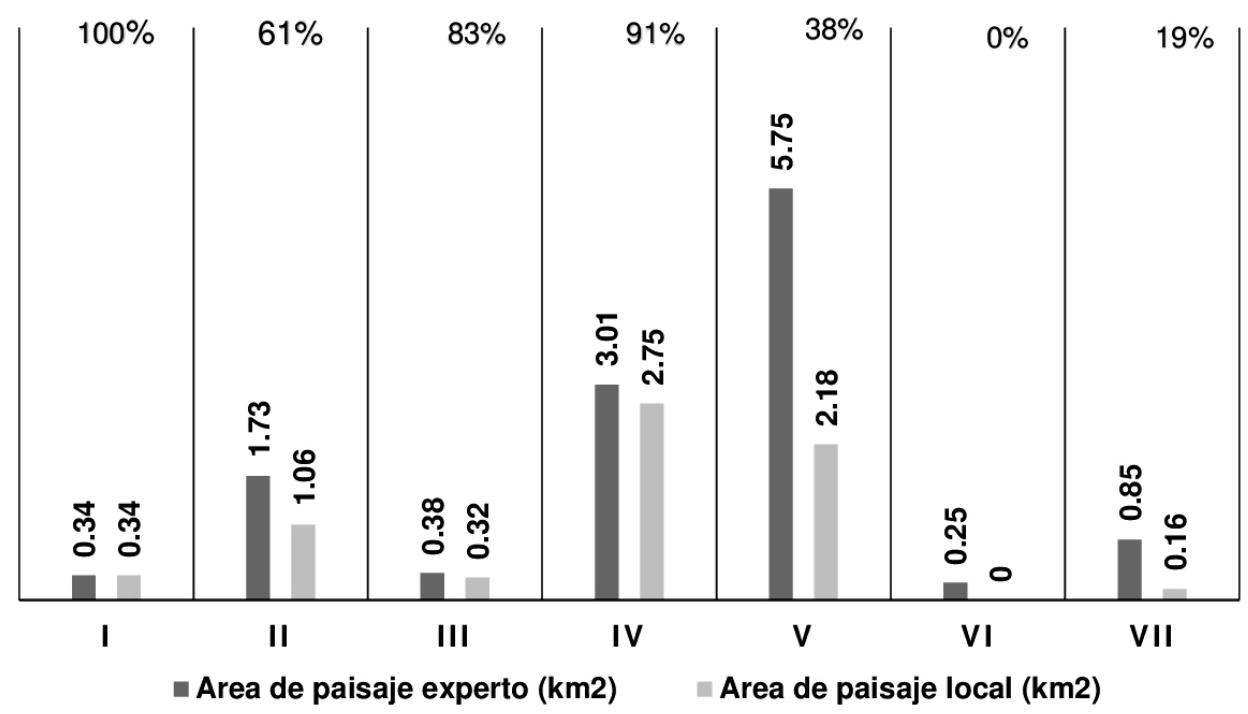

Fuente: Elaboración propia con base en los resultados obtenidos a partir de la cartografía de ambos enfoques.

Se observa que el reconocimiento y delimitación espacial de las unidades de paisaje por el conocimiento local coincidió en un $55.3 \%$ con la definición de polígonos del método experto de los paisajes físico-geográficos.

\section{Conclusiones}

Con el método empleado fue posible obtener un insumo cartográfico y además fue efectivo para el dialogo de saberes entre el conocimiento técnico y el empírico que tienen los ejidatarios en torno a los paisajes y a su caracterización. 
Al respecto de las características de los atributos en los paisajes, los resultados muestran que existe una relación directa entre los atributo descritos en ambos enfoques, sin embargo los contrastes entre ellos es conceptual, es decir, el enfoque experto se basa en un conocimiento occidental, donde cada aspectos físico y biológico del territorio es definido y validado por la tradición científica; en cambio, la interpretación de esos atributos del paisaje por los ejidatarios, están dados por un conocimiento tradicional, establecido por el valor utilitario del territorio, los cuales tienen una representatividad entre la misma comunidad y por lo tanto son localmente válidas.

De los resultados obtenidos, se concluye que en el enfoque experto, la geoforma fue el factor más importante para la distinción entre las unidades de paisajes; a diferencia de la interpretación local, donde el factor que tuvo mayor peso fue el uso del territorio derivado de la convivencia cotidiana de los lugareños con su entorno inmediato (traspatio, parcela, ejido, comunidad, etcétera).

Resultó factible poner en práctica las herramientas participativas (transecto, entrevista semiestructurada, mapa mental y SIGP) para facilitar la visualización y el entendimiento del mapa experto hecho en gabinete para la interpretación espacial de los ejidatarios y así traducir ese conocimiento local a un lenguaje cartográfico.

El uso de la prueba de bondad de ajuste resultó una herramienta estadística adecuada porque se observó el grado de adecuación de la interpretación de las unidades de paisaje por los ejidatarios con el modelo cartográfico experto. Así también, junto con el análisis de correspondencia espacial, los resultados refuerzan la evidencia que ambos enfoques tienen discrepancia espacial. Este resultado responde también a los diferentes esquemas de conceptuales entre ambos enfoques. 


\section{Referencias}

AAPYREFO-Asesores en aprovechamiento y restauración forestal, S.C. (2014). Reporte de evaluación rural participativa para el ejido Santa Cruz Ojo de Agua, Michoacán.

Aguirre L., R. (2010). Unidades campesinas de paisaje: estudio de caso en el ejido Nexpa, Michoacán, tesis de Maestría en Geografía, CIGAUNAM, Morelia, Michoacán.

Balram, S., Dragicevic, S., and Mederdith, T. (2004). A collaborative GIS method for integrating local and technical knowledge in establishing biodiversity conservation priorities. Biodiversity and Conservation 13:1195-1208.

Barajas A., A. G. (2012). Etnopedología en la reserva de la biosfera Zicuirán-Infiernillo, municipio de la Huacana, Michoacán, México. Tesis de Licenciatura. Universidad Michoacana de San Nicolás de Hidalgo, Morelia, Michoacán.

Barrera, B., N. (1988). Etnoedafología Purépecha: conocimiento y uso de los suelos en la cuenca de Pátzcuaro. México Indígena 24:47-52.

Barrera L., S. (2009). Reflexiones sobre Sistemas de Información Geográfica Participativos (SIGP) y cartografía social. Cuadernos de geografia 18: 9-23.

Bocco, G., Mendoza, M., Velázquez, A., \& Torres, A. (1999). La regionalización geomorfológica como una alternativa de regionalización ecológica en México. El caso de Michoacán de Ocampo. Investigaciones Geográficas 40: 7-22.

Bocco, G., Mendoza, M, Velázquez, A. (2001). Remote sensing and GIS based geomorphological mapping a tool for land use planning in developing countries. Geomorphology 39: 211-219.

Chalmers, N., and Fabricius, C., (2007). Expert and Generalist Local Knowledge about Land-cover Change on South Africa's Wild Coast: Can Local Ecological Knowledge Add Value to Science?. Ecology And Society 12: 10.

CONAFOR (2007). Manual básico para el Ordenamiento Territorial Comunitario (OTC), Comisiona Nacional Forestal.

Escalante, S, R. (2006). Desarrollo rural, regional y medio ambiente. ECONOMÍAunam, 3: 70-94 
Espinoza, M, A. (2013). Paisajes antropo-naturales en Tzintzuntzan y sus alrededores. Tesis de Maestría. Universidad Nacional Autónoma de Mexico, Mexico, D.F.

Fagerholm, N., Käyhkö, N., and Van Eetvelde, V. (2013). Landscape characterization integrating expert and local spatial knowledge of land and forest resources. Environmental Management 52:660-682.

García, A., E. (2004). Modificación al Sistema de Clasificación Climática de Köppen (para adaptarlo a las condiciones de la República Mexicana). Quinta edición. Instituto de Geografía-UNAM.

González, G, A., Bojórquez, J. I., Nájera, O., García, J. D., Madueño, A. \& Flores, F. (2009). Regionalización ecológica de la llanura costera norte de Nayarit, México. Investigaciones Geográficas 69: 21-32

Instituto Nacional de Estadística y Geografía (1973). Carta Geológica, clave F14C85 y F14C86 escala 1:50000. Recuperado de http://www. inegi.org.mx/inegi/contenidos/ProductosyServicios/default.aspx

Instituto Nacional de Estadística y Geografía (1974). Carta edafológica, clave F14C85 y F14C86 escala 1:50000. Recuperado de http://www. inegi.org.mx/inegi/contenidos/ProductosyServicios/default.aspx

Instituto Nacional de Estadística y Geografía (1974). Carta temática de Uso de Suelo y Vegetación, clave F14C85 y F14C86 escala 1:50000. Recuperado de http://www.inegi.org.mx/inegi/contenidos/ProductosyServicios/default.aspx

Instituto Nacional de Estadística y Geografía (2013). Modelo Digital de Elevación. Recuperado de http:/www.inegi.org.mx/geo/contenidos/ datosrelieve/continental/descarga.aspx

Instituto Nacional de Estadística y Geografía (2015). Carta topográfica con clave F14C85 y F14C86 escala 1:50000. Recuperado de http://www3. inegi.org. $\mathrm{mx} / \mathrm{sistemas} /$ biblioteca/ficha.aspx? upc $=702825203665$

Mateo, J. (2002). Geografía de los paisajes. Facultad de Geografía, Universidad de la Habana. 194 p.

McCall, M. K. (2011). Mapeando el territorio : paisaje local, conocimiento local, poder local. En Bocco, G., Urquijo, P., Vieyra, A. (Coord.) (2011). Geografía y Ambiente en América Latina (pp. 221-246). México. Centro de Investigaciones en Geografía Ambiental-UNAM. Negrete, G. \& Aguilar E (2006). Territorios Rurales, Política de Planeación y Ordenamiento Ecológico Local-Comunitario en México. En Anta, 
S., Arreola, A., González, M. y Acosta, J. (comps.), Semblanza histórica del Ordenamiento Ecológico Territorial en México, México DF. Negrete, G., \& Bocco, G. (2003). El ordenamiento ecológico comunitario : una alternativa de planeación participativa en el contexto de la política ambiental de México. Gaceta ecológica 68: 9-22

Ojeda, T. E., León, V. J. M., Michael, A., Dunn, C., Cajuste, B. L. (2015). Cartografía participativa para el Ordenamiento Territorial Comunitario: el caso del ejido de Santa María Nativitas, Texcoco, Estado de México. En Sorani, V., Alquicira, A. M. L. (eds.) Perspectivas del Ordenamiento Territorial Ecológico en América y Europa, México.

Pájaro, H, D. (2010). La cartografía de tierras: un contraste epistemológico. Revista de Geografía Agrícola, 9-23.

Pájaro, H, D., \& Tello, E. (2014). Fundamentos epistemológicos para la cartografía participativa. Etnoecológica 10: 1-20

Priego, S., A. G, Bocco, G., Mendoza, M., \& Garrido, A. (2010). Propuesta para la generación semiautomatizada de unidades de paisaje. Recuperado de http://www.ciga.unam.mx/publicaciones/index.php/ciga/ book/12-coleccionesciga/17-propuestas-para-la-generacion-semiautomatizada-de-unidades-de-paisaje

Roba, H., \& Oba, G. (2009). Community participatory landscape classification and biodiversity assessment and monitoring of grazing lands in northern Kenya. Journal of Environmental Management 90:673-682.

Sánchez, N, D. (2011). Potencial de los paisajes para actividades de turismo de naturaleza en el sector de la costa michoacana rio Coalcomanel farito. Tesis de Maestría. Universidad Nacional Autónoma de México, Morelia, Michoacán.

Soto, B., S. (2010). Diseño de una metodología de identificación y cartografía de unidades de paisaje a diferentes escalas. Aplicación al caso de Puerto Rico. Tesis doctoral. Universitat de Girona. Departament de Geografía. 
